\title{
Research on the SMA damping control of large-span spatial structures under multi-point input
}

\author{
Jianbo Dai, Xiyu Zhu \\ School of Mechanical Engineering, Xi'an Shiyou University, Xi'an, China \\ d_jianbo@foxmail.com,375209240@qq.com
}

\begin{abstract}
Keywords: Shape memory alloys; Large-span spatial structure; Multi-point input; Seismic control Abstract: Considering the adverse effects of the multi-point input of large-span spatial structure, the SMA damper bar was designed to replace original bars in the structure. In this paper, there were two projects to replace SMA damper bars, one was replaced of some lower chord's bars, The other was replaced of some web bars, and the effects of this two replacement situations were compared. The analysis has indicated that the lattice type was not changed and the reasonable control was done with this method. So it was a new and superior method of energy dissipation.
\end{abstract}

\section{Introduction}

For the engineering practice of large spatial reticulated structures, in addition to point out the necessity of the multi-point input, the structure response under multi-point seismic is more important for long-span spatial structures ${ }^{[1]}$. Analysis structure to produce the adverse factors of these reactions, and then take measures to make the necessary vibration control.Produce the adverse factors of these reactions on structure are analyzed, and then take measures to make the necessary vibration control.Therefore, for the targets of ensuring the architectural style of the large-span spatial structures and disaster-resistant reliability, using new intelligent materials-shape memory alloy (shape memory alloys, abbreviated SMA) ${ }^{[2]}$ material with unique mechanical properties of dynamic disaster protection designed for large-span spatial structure characteristics of SMA damper rod, and set it in the grid structure study on seismic response control of the replacing the original link.

\section{Under multi-point seismic response analysis of long-span spatial structures}

\section{Multi-point input equation of motion}

A linear elastic discrete element system with $\mathrm{N}$ degrees of freedom and $\mathrm{M}$ supporting,the equation can be written as follows ${ }^{[3]}$ :

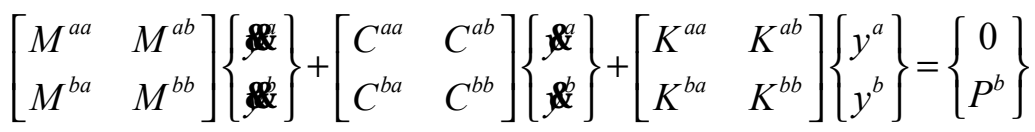

Where $\mathrm{M}$ is the structure supports quality matrix, $\mathrm{C}$ is the damping matrix of structure supports, $\mathrm{K}$ is the stiffness matrix of structure supports, $y^{a}$ is displacement vector of structure's non-bearing point degree of freedom. $y^{b}$ is displacement vector of structure's supporting point degree of freedom. Take a matrix that mark a matrix related to the supporting degree of freedom; Take the $b$ matrix representation and matrix about the supporting degree of freedom; Take the ab matrix representation structure of degrees of freedom and the supporting degree of freedom coupling matrix.

\section{Calculation model}

Large-span space frame structure with mesh pyramid and nodes for welded joints.Plane size for $108 \mathrm{mx} 72 \mathrm{~m}$, grid sizes $3 \mathrm{mx} 3 \mathrm{~m}, 2.9 \mathrm{~m}$ thick, every $36 \mathrm{~m}$ cloth a column and the column is $10 \mathrm{~m}$ tall. The material is Q235, the top chord for $\phi 168 \times 12$, the lower chord for $\phi 159 \times 10$, the web member for $\phi 133 \times 8$, the steel-pipe column for $\phi 900 \times 24$, as shown in Figure 1.The earthquake wave selects the EL-Centro wave, the computing time all takes is $10 \mathrm{~s}$, the time-gap is $0.02 \mathrm{~s}$.Presses 8 degrees after the EL-Centro wave often to meet the earthquake and rarely to meet the earthquake proportion amplitude modulation separately and so on acceleration peak value to carry on the level input, the most greatly 
horizontal acceleration peak value respectively is 70gal and 400gal.Analysis of the seismic response of the structure under consistent input and multi-point input respectively. Then select the bars with node displacement maximum and the shaft of the most to do the following:

$$
\alpha=\frac{\text { Peak response under multi - support seismic input }- \text { Peak response underconsistent seismic input }}{\text { Peak response }}
$$

Peak response underconsistent seismic input

Fig.1 Schematic diagram of grid structure calculation model

\section{The result of a calculation and analysis}

Table.1 Node displacement peak under earthquake of same input and multi-point input

\begin{tabular}{cccccc}
\hline Acceleration Peak/gal & Node & Direction & Same Input $/ \mathrm{mm}$ & Multi-Point Input $/ \mathrm{mm}$ & $\alpha$ \\
\hline \multirow{2}{*}{400} & \multirow{2}{*}{203} & $x$ & 24.5 & 29.7 & $21 \%$ \\
& & $y$ & 42.3 & 53.0 & $25 \%$ \\
\multirow{2}{*}{70} & \multirow{2}{*}{203} & $y$ & 5.6 & 7.2 & $29 \%$ \\
& & 9.9 & 3.3 & $34 \%$ \\
\hline
\end{tabular}

Table.2 Axial force peak of bars under earthquake of same input and multi-point input

\begin{tabular}{ccccc}
\hline Acceleration Peak/gal & Bars & Same Input $/ \mathrm{kN}$ & Multi-Point Input $/ \mathrm{kN}$ & $\alpha$ \\
\hline 400 & 3240 & 29.3 & 39.6 & $35 \%$ \\
70 & 3240 & 6.6 & 9.2 & $39 \%$ \\
\hline
\end{tabular}

The table 1 and table 2 shows that, compared with the consistent input, truss structures under multiple support excitation of EL-Centro wave with peak acceleration of $400 \mathrm{gal}$,x direction displacement has a $21 \%$ increase at most; $y$ direction displacement has a $25 \%$ increase at most; the member internal force has a $35 \%$ increase at most.Under the action of EL - Centro wave whose peak acceleration of 70 gal, $\mathrm{x}$ direction displacement has a $29 \%$ increase at most; $y$ direction displacement has a $34 \%$ increase at most; the member internal force has a $39 \%$ increase at most.Note whether under the rare occasions earthquake role or in multiple earthquake role, multi-point seismic response of structures with consistent input (such as displacements and internal forces of rod) are of great differences.So for large-span spatial structure, must consider the effect of the earthquake response of the structure of multi-point input, to ensure the safe and reliable of this kind of important structures.

\section{SMA damper replacement bars' vibration damper control analysis under multi-point input}

In this paper, the SMA damping bar is set up in the large span space truss structure as shown in Figure 1 , replacing some common members to dissipate the seismic energy so that the seismic response can be effectively controlled.The elastic modulus of SMA wire is $5.5 \mathrm{E}+10$, the Poisson's ratio is 0.3 , the density is $6450 \mathrm{~kg} / \mathrm{m} 3$, the damping ratio is about 0.02 . The alloy wire area of damper $\mathrm{A}=78.5 \mathrm{~mm}^{2}$.Introduce the following formula expression of damping effect:

$$
\varphi=\frac{\text { uncontrolled condition response peak value-condition response peak value under control }}{\text { uncontrolled condition response peak value }}
$$

\section{Layout and structure response of SMA damping bar}

In this paper, two kinds of SMA damping rod arrangement scheme are designed.The plan one is using the SMA damping bar arranged in every part of the grid bottom chord bar to replace the original layout, and the arrangement in Figure 2.The second scheme is that the SMA damping bar is arranged on each 
grid pole to replace the original structural member, and the arrangement is shown in figure 3. The time history curves of the two schemes are shown in Figure 4 and figure 5 respectively.

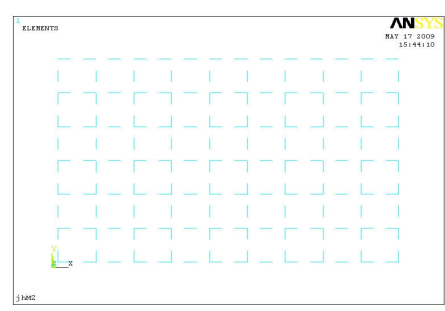

Fig. 2 project one of SMA damper bars

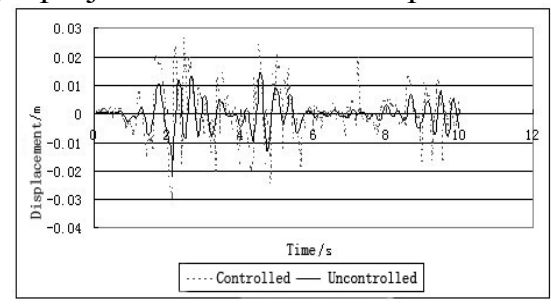

(a)

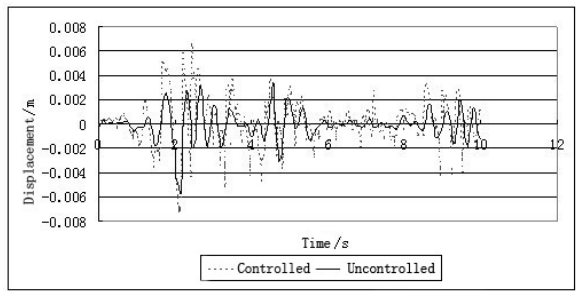

(c)

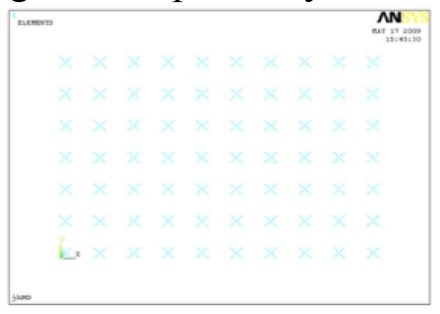

Fig. 3 project two of SMA damper bars

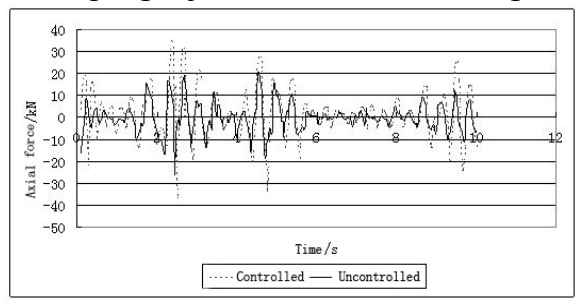

(b)

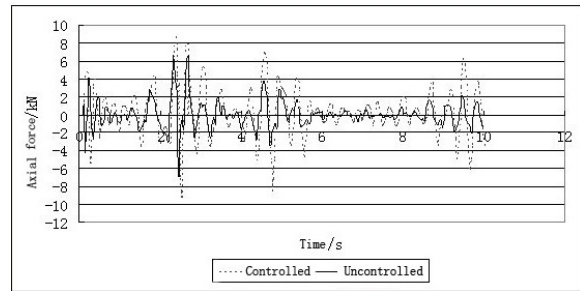

(d)

Fig.4 The controlled and uncontrolled time-history curve of $x$ - displacement of node 203 and axial force of bar 3240 under earthquake (project one)

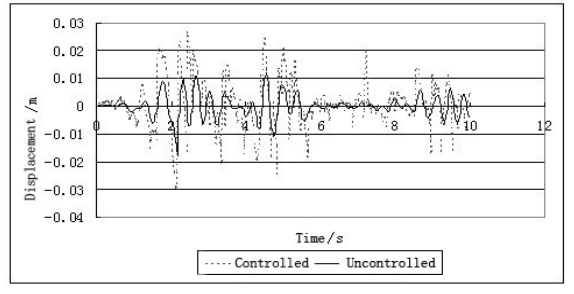

(a)

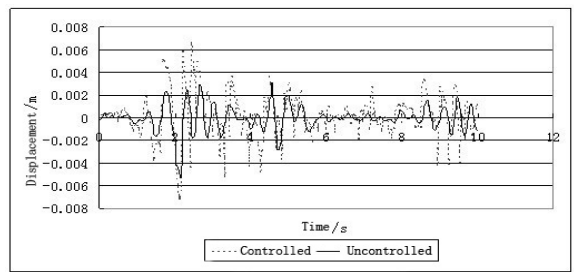

(c)

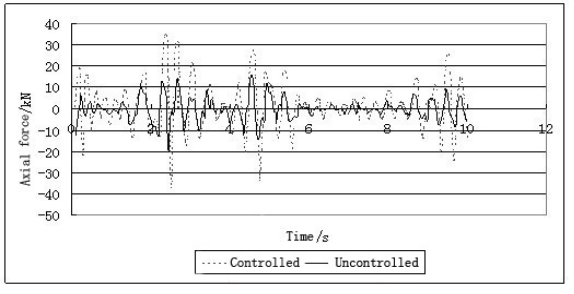

(b)

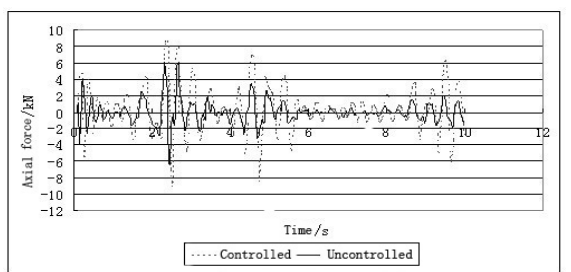

(d)

Fig. 5 The controlled and uncontrolled time-history curve of $\mathrm{x}$ - displacement of node 203 and axial force of bar

\section{Result analysis} 3240 under earthquake (project two)

Table. 3 The controlled effects of node displacement peak under earthquake

\begin{tabular}{cccccc}
\hline Project & Acceleration Peak/gal & Direction & Uncontrolled $/ \mathrm{mm}$ & Controlled $/ \mathrm{mm}$ & $\varphi$ \\
\hline \multirow{3}{*}{ Project one } & \multirow{2}{*}{400} & $x$ & 29.7 & 21.2 & $27 \%$ \\
& & $y$ & 53.0 & 32.3 & $39 \%$ \\
& \multirow{2}{*}{70} & $x$ & 7.2 & 5.7 & $20 \%$ \\
& \multirow{3}{*}{ Project two } & $y$ & 13.2 & 9.4 & $29 \%$ \\
& \multirow{2}{*}{70} & $x$ & 29.7 & 18.1 & $39 \%$ \\
& & $y$ & 53.0 & 26.9 & $49 \%$ \\
& & $x$ & 7.2 & 5.3 & $45 \%$ \\
& & $y$ & 13.2 & 8.5 & $26 \%$ \\
\hline
\end{tabular}


Table. 4 the controlled effects of axial force peak of 3240 bar under earthquake

\begin{tabular}{ccccc}
\hline Project & Acceleration Peak/gal & Uncontrolled $/ \mathrm{kN}$ & Controlled $/ \mathrm{kN}$ & $\varphi$ \\
\hline \multirow{2}{*}{ Project one } & 400 & 36.9 & 26.1 & $33 \%$ \\
& 70 & 9.2 & 7.4 & $21 \%$ \\
\multirow{2}{*}{ Project two } & 400 & 36.9 & 19.9 & $45 \%$ \\
& 70 & 9.2 & 6.5 & $26 \%$ \\
\hline
\end{tabular}

From table 3 and table 4, we can see that under the first control scheme, the space truss structure is under the action of EL-Centro wave with the peak acceleration of $400 \mathrm{gal}$,x direction displacement of damping effect has a $27 \%$ increase at most; $y$ direction displacement of damping effect has a $37 \%$ increase at most; the member internal force of damping effect has a 33\% increase at most.Under the action of EL-Centro wave with the peak acceleration of 70gal, $x$ direction displacement of damping effect has a $20 \%$ increase at most; y direction displacement of damping effect has a $29 \%$ increase at most; the member internal force of damping effect has a $21 \%$ increase at most.while under the second control scheme, the space truss structure is under the action of EL-Centro wave with the peak acceleration of $400 \mathrm{gal}, \mathrm{x}$ direction displacement of damping effect has a 39\% increase at most; $\mathrm{y}$ direction displacement of damping effect has a $49 \%$ increase at most; the member internal force of damping effect has a $45 \%$ increase at most.Under the action of EL-Centro wave with the peak acceleration of $70 \mathrm{gal}$, $\mathrm{x}$ direction displacement of damping effect has a $45 \%$ increase at most; $y$ direction displacement of damping effect has a $26 \%$ increase at most; the member internal force of damping effect has a $26 \%$ increase at most.

\section{Conclusion}

(1)The seismic response of long-span space structure of multi-point input and consistent input have great differences(such as displacement and internal force), so we must consider the response of multi-point input on the earthquake, in order to ensure the safety and reliability of this kind of structure.

(2)The two schemes of replacing the original member with the SMA damping bar for the energy dissipation control of the large span space structure are very obvious,so using the SMA damper bar to replace original member of the spatial grid structure is a kind of very good damping control method. (3)Compared with the two control schemes, damping effect of the second control scheme to replace the web member is more obvious than the first to replace the bottom chord.So the second control scheme can be used for the optimization design of this kind of structure.

\section{Acknowledgements}

This work was financially supported by the grants from National Natural Science Found Project of China (51405385); Special Research Project in Shaanxi Province Department of Education (15JK1562); Key Laboratory Project of Shaanxi Department of Education by Visiting Scholars (14JS045); Youth Science and Technology Innovation Fund Project of Xi'an Shiyou University(2013BS024).

\section{References}

[1] S. Kato, S.Nakazawa and L.Su. Effects of wave passage and local site on seismic responses of a large-span reticular dome structure. Steel Construction Engineering,Vol. 10 (2003), p.91-106

[2] S.L.Wang, S.G.Ju, S.Q.Su. The behavior of dynamic response of shape memory alloy and its application in vibration control. J.Xi'an Univ. of Arch.\& Tech.(Natural Science Edition),Vol. 31(1999), p.14-17

[3] E.J. Graesser, A. Cozzarellif. Shape memory alloys as a new material for aseismic isolation. J. of Eng. Mech, Vol. 11(1991), p.2590-2608 